\title{
Estudio Morfométrico del Hígado de Ratón en la Enfermedad de Chagas Experimental
}

\author{
Morphometrical Study of the Mouse Liver in the Experimental Chagas' Disease \\ "Louise Helena Olhan Ragonha; *Ana Amélia Carraro Abrahão; **Miguel Angel Sala; ${ }^{* *, * * * * R u b e r v a l ~ A . ~ L o p e s ; ~}$ \\ ${ }^{* *, * * * *}$ Rosa Domingues Ribeiro; *José Clovis do Prado Jr.; "Sérgio de Albuquerque \& ${ }^{* * * *}$ Sérgio Zucoloto.
}

RAgOnha, L. H. O.; ABRAhão, A. A. C.; SAlA, M. A.; LOPES, R. A.; RIBEIRO, R. D.; PRADO JR., J. C.; ALBUQUERQUE, S. \& ZUCOLOTO, S. Estudio morfométrico del hígado de ratón en la enfermedad de Chagas experimental. Int. J. Morphol., 24(3):383-390, 2006.

RESUMEN: El objetivo de este trabajo fue caracterizar histopatológicamente y morfométricamente las alteraciones del tejido hepático de ratón, durante la fase aguda de la infección por la cepa MORC-2 de Trypanosoma cruzi. Esta cepa mostró acentuado tropismo por el hígado, con numerosos nidos de amastigotes en los cortes examinados. El hígado de los animales infectados estaba constituido por células menores, con citoplasma granuloso. En algunas áreas, los sinusoides estaban congestionados y las células de Kupffer hipertróficas e hiperplásicas. El tejido hepático mostró focos circunscritos de células inflamatorias en áreas de necrosis, sinusoides, en torno de las venas centrolobulillares y de los espacios porta. La vena centrolobulillar estaba dilatada y congestionada, con necrosis focales y ruptura de la pared en algunos campos. Los espacios porta estaban desorganizados, a veces, con intenso infiltrado inflamatorio. En algunas áreas fue posible observar degeneración cística (spongis hepatis). Por todo el tejido hepático se observaron nidos de amastigotes, de tamaño variable, algunos rodeados por infiltrado inflamatorio crónico. En el espacio porta, el volumen relativo de los conductos biliares y vasos sanguíneos, así como la densidad de superficie de las arterias fueron mayores en el grupo infectado.

PALABRAS CLAVE: Morfometría; Histopatología; Hígado; Ratón; Trypanosoma cruzi; Cepa MORC-2.

\section{INTRODUCCIÓN}

El Trypanosoma cruzi, agente etiológico de la tripanosomiasis latinoamericana, es responsable de una grave enfermedad, de gran importancia en muchos países da América Latina. Según la Organización Mundial de la Salud, existen 16 a 18 millones de personas infectadas en las Américas y 80 a 100 millones de personas expuestas al riesgo de infección (WHO, 1997; Pinto et al., 1996). La transmisión de la enfermedad ocurre principalmente por un vector (80 a 90\%), transfusión sanguínea (5 a 20\%) y ruta congénita $(0.5$ a $8 \%)$ (Días, 2000).

Básicamente, las alteraciones de los tejidos predominantes en la enfermedad de Chagas dependen del tropismo de la cepa y, en consecuencia, del mayor grado de parasitismo de los diferentes órganos del huésped infectado. Andrade (1974, 1985), estudiando diferentes cepas de Trypanosoma cruzi las clasificó en tres tipos o biodemas, de acuerdo con las características biológicas e histopatológicas.
De este modo, en la infección con las cepas del Tipo I, se observa, en las fases iniciales, hasta 10 días post-infección, un intenso parasitismo de macrófagos, principalmente en órganos como bazo, hígado, ganglios linfáticos y tejido conjuntivo intersticial. En la infección con las cepas del Tipo II, cuya parasitemia presenta curso más lento, entre el $12^{\circ} \mathrm{y}$ $20^{\circ}$ día, las lesiones predominantes en la fase aguda ocurren en el miocardio, produciendo también, en menor grado, lesiones de músculos esqueléticos y lisos, con compromiso de los plexos nerviosos mioentéricos.

Varios trabajos muestran que las cepas de $T$. cruzi son poblaciones complejas que difieren en sus características morfológicas, biológicas, genéticas, así como en el comportamiento de la infección en el huésped vertebrado. El tropismo por diferentes tejidos también ha sido referido en diversos estudios que procuran diferenciar las poblaciones y algunas presentan, en la fase inicial de la infección, un in-

\footnotetext{
* Facultad de Ciencias Farmacéuticas de Ribeirão Preto, USP, Brasil.

** Facultad de Odontología de Ribeirão Preto, USP, Brasil.

${ }^{* * *}$ UNIFRAN, Brasil.

${ }^{* * * * *}$ Facultad de Medicina de Ribeirão Preto, USP, Brasil.
} 
tenso parasitismo de macrófagos, principalmente en órganos como bazo, hígado y ganglios linfáticos.

La cepa MORC-2, aislada del murciélago Phyllostomus h. hastatus, mostró ser patogénica para el ratón y presentó un intenso tropismo por el hígado. De este modo, el objetivo del presente estudio fue evaluar las alteraciones histopatológicas causadas por la cepa MORC-2 en el hígado de ratones, en la fase aguda de la infección.

\section{MATERIAL Y MÉTODO}

Cepa de Trypanosoma cruzi. Fue utilizada la cepa MORC2, aislada del murciélago Phyllostomus h. hastatus capturado en la región de Ribeirão Preto, Estado de San Pablo, Brasil (Albuquerque et al., 1992). Esta cepa, clasificada como T. cruzi II (Dost et al. 2002), es mantenida en ratones albinos, variedad Swiss, en el Laboratorio de Parasitología de la Facultad de Ciencias Farmacéuticas de Ribeirão Preto, Brasil, mediante subinoculación intraperitoneal de sangre, con intervalos de 10 días. Morfológicamente, se caracteriza por predominio de formas intermediarias y finas, con niveles máximos de parasitemia en torno del $10^{\circ}$ día post-inóculo.

Animales. Fueron utilizados ratones blancos (Mus musculus), variedad Swiss, machos, adultos jóvenes, pesando aproximadamente $23 \mathrm{~g}$ y divididos en dos grupos:

Grupo infectado: constituido por 10 animales inoculados intraperitonealmente con $1 \times 10^{5}$ tripomastigotes sanguíneos de la cepa MORC-2 de T. cruzi.

Grupo control: constituido por 10 animales inyectados con suero fisiológico.

Todos los animales fueron mantenidos en condiciones controladas de luz (12 h-luz, 12 h-oscuridad), temperatura $\left(25^{\circ} \pm 2^{\circ} \mathrm{C}\right)$ y humedad $(55 \pm 10 \%)$, y alimentados con ración comercial y agua ad libitum.

Técnica de disección. En el $10^{\circ}$ día de la infección, los animales fueron anestesiados con una inyección intraperitoneal de tribromoetanol $(0,1 \mathrm{~mL} / 10 \mathrm{~g}$ de peso corporal de una solución al 2,5\% en $\mathrm{NaCl} 0,15 \mathrm{M}$ ) y luego de abertura amplia del abdomen, fue retirado el hígado de los animales de los dos grupos experimentales. Fragmentos de los hígados fueron fijados en una solución de alcohol $80 \%$ - $85 \mathrm{ml}$, formol - $10 \mathrm{ml}$ y ácido acético - $5 \mathrm{ml}$ (ALFAC), durante $24 \mathrm{~h}$.
Técnica morfométrica. Los tejidos, fijados e incluidos en parafina fueron cortados de forma seriada, con $6 \mu \mathrm{m}$ de espesor, y posteriormente teñidos con hematoxilina y eosina.

Cariometría. Fue realizado el estudio cariométrico de los hepatocitos en la región de la vena centrolobulillar, en la región del espacio porta y en áreas al azar de la región intermediaria del lobulillo hepático. Fueron analizados 50 núcleos por animal en cada región, estimándose los siguientes parámetros nucleares (Sala et al., 1994): diámetro mayor, diámetro menor, diámetro medio, relación entre diámetro mayor y diámetro menor, volumen, área, perímetro, relación volumen/área, coeficiente de forma, índice de contorno y excentricidad.

Estereología. Mediante la utilización del retículo de 100 puntos (Merz, 1968), fueron estimados los volúmenes relativos de hepatocitos, sinusoides, conductos biliares, vasos y células inflamatorias en el hígado de los dos grupos de animales, en 20 campos microscópicos de cada animal (Chalkley, 1943; Weibel, 1969).

Para avaluar las alteraciones tisulares fueron estimados los siguientes parámetros de los hepatocitos: volumen relativo nuclear, volumen relativo citoplasmático, relación núcleo-citoplasma, volumen celular medio, volumen citoplasmático medio y densidad numérica de los hepatocitos (Sala et al., 1992). La densidad de superficie, el diámetro medio y el espesor de la pared de los conductos biliares y vasos del espacio porta fueron estimados mediante los métodos de Tomkeieff (1945), Sala et al. (1980) y Sala et al. (1981), respectivamente.

Análisis estadístico. Los resultados obtenidos fueron analizados estadísticamente mediante la prueba no paramétrica de Wilcoxon-Mann-Whitney (Sprent \& Smeeton, 2001).

\section{RESULTADOS}

Histopatología del hígado de ratones infectados. El hígado de los animales infectados se mostró constituido por hepatocitos poco voluminosos, con citoplasma granuloso presentando nidos de amastigotes (Fig. 1A). Los núcleos poseían tamaños variables, siendo algunos gigantes e irregulares. Fueron observados, con frecuencia, hepatocitos en mitosis y, también, algunos hepatocitos binucleares. En algunas áreas, los sinusoides estaban dilatados y las células de Kupffer hipertróficas e hiperplásicas (Fig. 1B). En todo el hígado eran visibles focos circunscritos de células inflamatorias, con predominio de mononucleares en los 

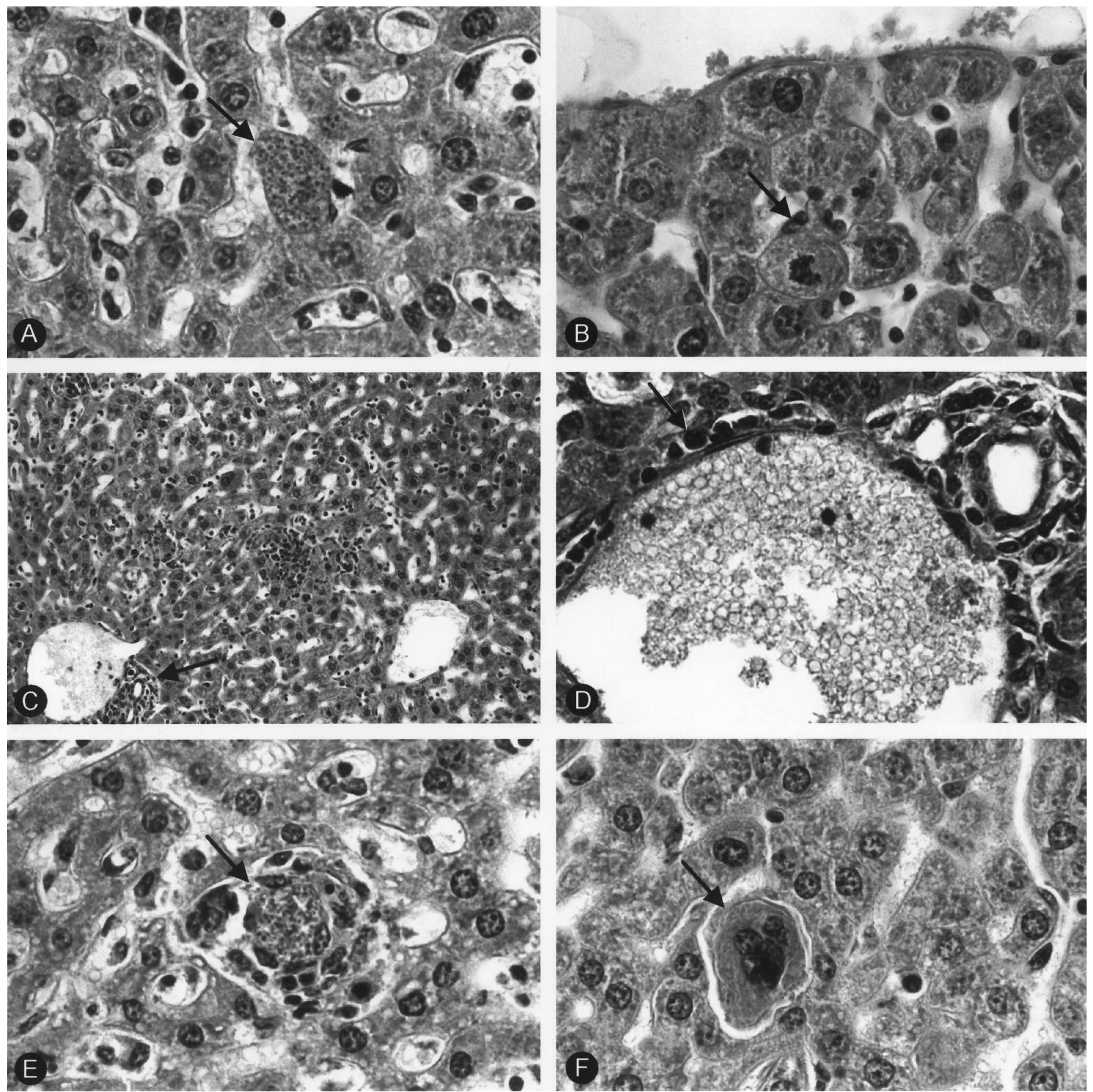

Fig. 1. Cortes histológicos de hígado de ratón infectado por la cepa MORC-2 de T. cruzi. HE. A - Nido de amastigotes (950X). B Hepatocitos pequeños, con citoplasma granuloso; sinusoides dilatados con células de Kupffer prominentes (950X). C - Conducto biliar con infiltrado inflamatorio (225X). D - Endotelitis de ramos de la vena porta junto al espacio porta y a la vena centrolobulillar (950X). E - Nido de amastigotes, rodeado de células inflamatorias crónicas (950X). F - Presencia de megacariocito (950X).

sinusoides y en los focos de necrosis del parénquima hepático. Estas células inflamatorias también eran visibles alrededor de las venas centrolobulillares y de los espacios porta, caracterizando un cuadro de endotelitis (Figs. 1C y D). La vena centrolobulillar estaba dilatada y congestionada, con necrosis focales y ruptura de la pared vascular en algunos campos microscópicos. Los espacios porta se presentaban desorganizados, algunos de ellos intensamente infiltrados con células inflamatorias. En algunas áreas fue posible observar degeneración cística (spongis hepatis). Por todo el tejido se encontraban nidos de amastigotes de tamaño variable, algunos de los cuales rodeados por numerosas células inflamatorias de tipo crónico (Fig. 1E). También fue observada la presencia de megacariocitos (Fig. 1F). 
Morfometría del hígado de ratones infectados. Los resultados del estudio morfométrico son mostrados en forma de Tablas. Así, los parámetros nucleares de los hepatocitos, en las diferentes regiones del lobulillo hepático, son presentados en la Tabla I, los parámetros estereológicos del lobulillo hepático son mostrados en las Tablas II y III, mientras que los parámetros correspondientes a vasos sanguíneos y conductos biliares de la región del espacio porta se encuentran en las Tablas IV y V.

Tabla I. Parámetros nucleares de los hepatocitos de la región del espacio porta, de la región pericentrolobulillar y de regiones aleatorias de áreas intermediarias del lobulillo hepático de ratones del grupo control (C) y del grupo infectado por la cepa MORC-2 de T. cruzi (I). Prueba de Wilcoxon-Mann-Whitney.

\begin{tabular}{|c|c|c|c|c|c|c|}
\hline \multirow{3}{*}{ Parámetros nucleares } & \multicolumn{6}{|c|}{ Región } \\
\hline & \multicolumn{2}{|c|}{ Intermediaria } & \multicolumn{2}{|c|}{ Espacio porta } & \multicolumn{2}{|c|}{ Pericentrolobulil lar } \\
\hline & $\mathrm{C}$ & I & $\mathrm{C}$ & I & $\mathrm{C}$ & I \\
\hline Diámetro mayor $(\mu \mathrm{m})$ & 8,89 & 9,13 & 7,66 & $9,26^{*}$ & 8,35 & 8,59 \\
\hline Diámetro menor $(\mu \mathrm{m})$ & 7,88 & 8,20 & 6,88 & $8,22 *$ & 7,45 & 7,65 \\
\hline Diámetro medio $(\mu \mathrm{m})$ & 8,36 & 8,65 & 7,26 & $8,72^{*}$ & 7,88 & 8,10 \\
\hline Relación D/d & 1,13 & 1,12 & 1,12 & 1,13 & 1,13 & 1,13 \\
\hline Volumen $\left(\mu \mathrm{m}^{3}\right)$ & 323,34 & 358,82 & 210,06 & $367,94^{*}$ & 268,23 & 301,10 \\
\hline Área $\left(\mu \mathrm{m}^{2}\right)$ & 55,90 & 59,88 & 42,03 & $60,90^{*}$ & 49,52 & 52,87 \\
\hline Perímetro $(\mu \mathrm{m})$ & 26,38 & 27,26 & 22,87 & $27,50^{*}$ & 24,86 & 25,54 \\
\hline Relación V/A & 5,57 & 5,76 & 4,84 & $5,81^{*}$ & 5,26 & 5,39 \\
\hline Coeficiente de forma & 0,99 & 0,99 & 0,99 & 0,99 & 0,99 & 0,99 \\
\hline Índice de contomo & 3,56 & 3,56 & 3,56 & 3,56 & 3,56 & 3,56 \\
\hline Excentricidad & 0,39 & 0,38 & 0,38 & 0,40 & 0,39 & 0,38 \\
\hline
\end{tabular}

(*) $\mathrm{p}<0,01$

Tabla II. Valores medios del volumen relativo (\%) de hepatocitos, sinusoides, conductos biliares, vasos y células inflamatorias de ratones del grupo control y del grupo infectado por la cepa MORC-2 de T. cruzi. Prueba de Wilcoxon-Mann-Whitney.

\begin{tabular}{lcc}
\hline \multicolumn{1}{c}{ Estructuras } & Grupo control & Grupo infectado \\
\hline Hepatocitos (\%) & 76,17 & $65,77^{* *}$ \\
Sinusoi des (\%) & 18,30 & $13,90^{*}$ \\
Conductos y vasos (\%) & 2,50 & $4,75^{* *}$ \\
Células inflamatorias (\%) & 2,97 & $15,58^{* *}$ \\
\hline
\end{tabular}

(*) $\mathrm{p}<0,05 \quad(* *) \mathrm{p}<0,01$
Tabla III. Parámetros estereológicos de los hepatocitos de ratones del grupo controle (C) e infectados por la cepa MORC-2 de T. cruzi (I). Prueba de Wilcoxon-Mann-Whitney.

\begin{tabular}{lcc}
\hline Parámetros estudiados & Grupo & Grupo \\
& control & infectado \\
\hline Volumen relativo nuclear $(\%)$ & 7 & 8 \\
Volumen relativo de citoplasma $(\%)$ & 93 & 92 \\
Relación núcleo/citoplasma & 0,0787 & 0,0878 \\
Volumen celular $\left(\mu \mathrm{m}^{3}\right)$ & 4262,06 & 4223,88 \\
Volumen de citoplasma $\left(\mu \mathrm{m}^{3}\right)$ & 3965,14 & 3884,26 \\
Densidad numérica $\left(\mathrm{N} / \mathrm{mm}^{3}\right)$ & 240330,70 & 239868,30
\end{tabular}


Tabla IV. Parámetros estereológicos de las arterias y venas de la región del espacio porta de ratones del grupo control (C) y del grupo infectado por la cepa MORC-2 de T. cruzi (I). Prueba de Wilcoxon-Mann-Whitney.

\begin{tabular}{lcccccc}
\hline & \multicolumn{3}{c}{ Arterias } & & \multicolumn{3}{c}{ Venas } \\
\cline { 2 - 3 } \cline { 5 - 6 } Parámetros & C & I & & C & I \\
\hline Densidad de volumen & 0,0012 & $0,0026^{*}$ & & 0,0547 & 0,0712 \\
Densidad de superficie $\left(\mathrm{mm}^{2} / \mathrm{mm}^{3}\right)$ & 2,70 & $3,80^{*}$ & & 21,40 & 24,10 \\
Diámetro medio $(\mu \mathrm{m})$ & 18,90 & $27,10^{*}$ & & 101,50 & 116,20 \\
\hline
\end{tabular}

$(*) \mathrm{p}<0,05$

Tabla V. Parámetros estereológicos de los conductos biliares de la región del espacio porta de ratones del grupo control y del grupo infectado por la cepa MORC-2 de T. cruzi. Prueba de Wilcoxon-Mann-Whitney.

\begin{tabular}{lcc}
\hline Parámetros & Grupo control & Grupo infectado \\
\hline Densidad de volumen & 0,0048 & 0,0050 \\
Densidad de superficie $\left(\mathrm{mm}^{2} / \mathrm{mm}^{3}\right)$ & 9,98 & 10,7 \\
Diámetro medio $(\mu \mathrm{m})$ & 19,3 & 19,1 \\
Espesor de la pared $(\mu \mathrm{m})$ & 4,34 & 5,89 \\
Diámetro de la luz $(\mu \mathrm{m})$ & 16,46 & 13,23 \\
\hline
\end{tabular}

\section{DISCUSIÓN}

Diversos trabajos han mostrado que los procesos patogénicos de las lesiones en la fase aguda de la infección chagásica en el ratón, son complejos e innumerables factores están involucrados, como el linaje de la cepa infectante, su multiplicación y muerte intracelular, la morfología del tripomastigote sanguíneo y su tropismo, así como los mecanismos inmunológicos humorales y celulares, causando las lesiones características de esa fase (Brener, 1965; Andrade et al., 1970, 1985a, 1985b; Silva et al., 1985; Ribeiro dos Santos et al., 1991; Brener \& Gazzinelli, 1997).

El T. cruzi tiene capacidad de invadir y multiplicarse dentro de diferentes células huésped, como macrófagos, fibras musculares lisas y estriadas, fibroblastos y aún mismo neuronas (Díaz-Limay et al., 2004). El acometimiento del hígado en la fase aguda de la infección chagásica ha sido comprobado en el Hombre y en animales infectados experimentalmente (Melo \& Brener, 1978). La cepa silvestre MORC-2, aislada del murciélago $P$. h. hastaus, presentó acentuado tropismo por el hígado de ratones, demostrándose numerosos nidos de amastigotes en los cortes histológicos examinados. En el espacio porta, el volumen relativo de los conductos biliares y de los vasos sanguíneos fue mayor en el grupo infectado, además de presentar aumento de la densidad de volumen y de superficie de las arterias. En las venas y conductos biliares no se observaron alteraciones de los parámetros estereológicos. Valores semejantes fueron demostrados en los hepatocitos de los dos grupos estudiados, para volumen citoplasmático, volumen celular, relación núcleo-citoplasma y número de células por milímetro cúbico.

Los núcleos se mostraron semejantes en los dos grupos de animales, en las regiones intermediarias y centrolobulillar. En la región alrededor del espacio porta, los núcleos se presentaron mayores, confirmado por los valores aumentados determinados cariométricamente para los diámetros mayor, menor y medio, volumen, área, perímetro y relación volumen/área. No se observó alteración de forma en los núcleos de los hepatocitos, en las tres regiones. Los sinusoides presentaron disminución de los volúmenes relativos. 
Las relaciones parásito-huésped en la infección de vertebrados por T. cruzi son caracterizadas por la multiplicación intracelular y evolución de las formas parasitarias, por las alteraciones de las células parasitadas y por la respuesta inmunológica, con su componente inflamatorio (Andrade, 2000). La reacción inicial al T. cruzi es la inflamación focal mononuclear debida a la ruptura de las células parasitadas. En algunos días puede ser detectada la presencia de complejos inmunes en el suero, junto con necrosis en los focos inflamatorios (Andrade, 1999).

Ratones infectados por las cepas Cali, Bolivia e Y de $T$. cruzi también presentaron lesiones inflamatorias en el hígado (Diego et al., 1991). En el presente trabajo, el examen microscópico de los cortes histológicos de hígado de ratones infectados por la cepa MORC-2, permitió observar que, en las regiones donde no había parasitismo ni proceso inflamatorio intenso, los hepatocitos estaban, en su mayoría, preservados.

Aspectos ultraestruturales de las lesiones hepáticas causadas por la cepa Bertoldo de T. cruzi, en ratones albinos, en la fase aguda de la infección, fueron relatados por Sanabria (1971). Fueron encontradas formas tripomastigotes en los sinusoides hepáticos y nidos de amastigotes en el citoplasma de las células de Kupffer, pero no se detectaron parásitos en los hepatocitos.

El examen histopatológico de ratones infectados por la cepa JALGO de T. cruzi demostró, durante la fase aguda, mayor tropismo por los músculos cardíaco y esquelético del ratón y menor tropismo por otros órganos. El cuadro histopatológico se caracterizó por la presencia de infiltrado celular en todos los órganos (Vera-Cruz et al., 2003). La cepa MORC-2 presentó tropismo por corazón e hígado de ratones, provocando intenso parasitismo, lesiones inflamatorias focales y muerte de los animales en la fase aguda de la infección. Los nidos de amastigotes estaban ampliamente distribuidos por el tejido hepático, sin ocurrir concentración en regiones específicas. En las regiones parasitadas fue observado intenso proceso inflamatorio, alrededor de los nidos de amastigotes y también en otras regiones, particularmente alrededor del espacio porta, hecho confirmado por el aumento del volumen relativo de las células inflamatorias, determinado estereólogicamente.

El T. cruzi manifiesta heterogeneidad genética, lo que explicaría los diferentes grados de tropismo por órganos diferentes (Macedo \& Pena, 1998). Los aspectos genéticos de los parásitos juegan un importante papel en determinar cuáles tejidos del huésped serán infectados y así ejercer una fuerte influencia en la patogénesis de la enfermedad de Chagas (Macedo et al., 2002, 2004). Ciertamente, el fenómeno del tropismo tisular diferencial es un efecto de las interacciones entre macromoléculas sobre la superficie, tanto del parásito como de las células huésped. Muchos antígenos de superficie del parásito han sido descritos con importantes moléculas involucradas en la invasión, pero que se conoce muy poco en relación a la interacción parásito-huésped.

\section{AGRADECIMIENTOS}

$\mathrm{Al} \mathrm{CNPq} \mathrm{por} \mathrm{la} \mathrm{beca} \mathrm{de} \mathrm{Iniciación} \mathrm{Científica} \mathrm{(PIBIC/}$ $\mathrm{CNPq}$ ) concedida a la primera autora, y al Sr. Antonio de Campos de la Facultad de Odontología de Ribeirão PretoUSP, Brasil, por la confección de las láminas histológicas.

RAGONHA, L.H.O.; ABRAHÃO, A.A.C.; SALA, M.A.; LOPES, R.A.; RIBEIRO, R.D.; PRADO JR., J.C.; ALBUQUERQUE, S. \& ZUCOLOTO, S. Morphometrical study of the mouse liver in the experimental Chagas' disease.Int. J. Morphol., 24(3):383-390, 2006.

SUMMARY: The objective of this work was to characterize histopatologically and morphometrically the alterations of the mouse liver during the acute infection by the MORC-2 strain of Trypanosoma cruzi. This strain showed marked tropism by the liver, with numerous nests of amastigotes in the examined sections. The liver of the infected animals was constituted by smaller cells, with granular cytoplasm. In some areas, the sinusoids were congested and the Kuppfer cells were hipertrofied and hiperplasic. The hepatic tissue showed circumscribed foci of inflammatory cells into necrotic areas, sinusoids, around the contrilobular veins and the portal spaces. The centrilobular vein was dilated and congested, with focal necrosis and rupture of the wall in some regions. The portal spaces were disorganized, sometimes with intense inflammatory infiltrate. In some areas it was possible to observe cystic degeneration (spongis hepatis). In the hepatic tissue, nests of amastigotes, of variable sizes, were observed, some surrounded by chronic inflammatory infiltrate. In the portal space, the relative volume of the biliary ducts and blood vessels, as well as the surface density of the arteries was greater in the infected group.

KEY WORDS: Morphometry; Histopathology; Liver; Mouse; Trypanosoma cruzi; MORC-2 Strain. 


\section{REFERENCIAS BIBLIOGRÁFICAS}

Albuquerque, S.; Ribeiro, R. D.; Carraro, A. A.; Lopes, R. A.; Prado Júnior, J. C.; Garcia, T. A. R. \& Toldo, M. P. A. Contribuição para o estudo da cepa de Trypanosoma cruzi isolada do morcego Phyllostomus h. hastatus. Rev. Esc. Farm. Odont. Alfenas, 14:3-8, 1992.

Andrade, S. G. Caracterização de cepas do Trypanosoma cruzi isoladas no Recôncavo Baiano. Rev. Pat. Trop., 1: 65-121, 1974

Andrade, S. G. Morphological and behavioural characterization of Trypanosoma cruzi strains. Rev. Soc. Bras. Med. Trop., 18:39-46, 1985.

Andrade, S.G. Patologia experimental da doença de Chagas. In: Brener, Z., Andrade, Z.A. \& Barral-Netto, M.T. (ed.) Trypanosoma cruzi e doença de Chagas, $2^{\mathrm{a}}$ ed. Rio de Janeiro, Guanabara-Koogan, 2000. pp. 186-200.

Andrade, S.G.; Carvalho, M.L. \& Figueira, R.M. Caracterização morfobiológica e histopatológica de diferentes cepas do Trypanosoma cruzi. Gaz. Méd. Bahia, $70: 32-42,1970$.

Andrade, V.; Andrade, S. G.; Barral Netto, M.T.; Pontes, A. L. \& Castro, R. Avaliação do comportamento de diferentes cepas do Trypanosoma cruzi na infecção de seis linhagens isogênicas de camundongos. Rev. Soc. Bras. Med. Trop., 18:143-54, 1985a.

Andrade, V.; Barral Netto, M.T. \& Andrade, S.G. Patterns of resistance of inbred mice to Trypanosoma cruzi are determined by parasite strain. Braz. J. Med. Biol. Res., 18:499-506, 1985b.

Andrade, Z. \& Andrade, S.G. Pathology and pathogenesis of Chagas' disease. In: Gilles, H. M. (ed). Protozoal diseases. London, Arnold, 1999.

Brener, Z. \& Gazzinelli, R.T. Immunological control of Trypanosoma cruzi infection and pathogenesis of Chagas' disease. Int. Arch. Allergy Immunol., 114:103$10,1997$.

Brener, Z. Comparative studies of different strains of Trypanosoma cruzi. Ann. Trop. Med. Parasitol., 59:1926, 1965.

Chalkley, H.W. Method for the quantitative morphologic analysis of tissues. J. Natl. Cancer Inst., 4: 47-53, 1943.
Dias, J.C.P. Epidemiologia. In: Brener, Z.; Andrade, Z. \& Barral-Netto, M.T. Trypanosoma cruzi e doença de Chagas. 2a. ed. Rio de Janeiro, Guanabara Koogan, 2000. 48-74.

Díaz-Limay, E.; Escalante, H. \& Jara, C.A. Niveles de parasitemia y alteraciones histopatológicas en Mus musculus BALB/c infectado con Trypanosoma cruzi obtenido de Panstrongylus chinai del Valle Chamán, La Libertad - Perú. Parasitol. Latinoam., 59:153-8, 2004.

Diego, J.A.; Penin, P.; Del Rey, J.; Mayer, R. \& Gamallo, C. A comparative pathological study of three strains of Trypanosoma cruzi in an experimental model. Histol. Histopathol., 6:199-206, 1991.

Dost, C. K.; Albuquerque, S.; Hemleben, V.; Engels, W. \& Prado Jr., J. C. Molecular genetic characterization of different Trypanosoma cruzi strains and comparison of their development in Mus musculus and Calomys callosus. Parasitol. Res., 88:609-16, 2002.

Macedo, A. M. \& Pena, S. D. J. Genetic variability of Trypanosoma cruzi: implications for the pathogenesis of Chagas' disease. Parasitol. Today, 14:119-24, 1998.

Macedo, A. M.; Oliveira, R. P. \& Pena, S. D. J. Chagas' disease: role of parasite genetic variation in pathogenesis. Exp. Rev. Mol. Med., 4:1-16, 2002.

Macedo, A. M.; Machado, C. R.; Oliveira, R. P. \& Pena, S. D. Trypanosoma cruzi: genetic structure of populations and relevance of genetic variability to the pathogenesis of Chagas' disease. Mem. Inst. Oswaldo Cruz, 99:1-12, 2004.

Melo, R.C. \& Brener, Z. Tissue tropism of different Trypanosoma cruzi strains. J. Parasitol., 64:475-82, 1978.

Merz, W. A. Streckenmessung an gerichteten Strukturen im Mikroskop und Anwendung zur Bestimmung von Oberflächen volumen Relationen im Knochengewebe. Mikroskopie, 22:132-42, 1968.

Pinto, J. C. Las enfermedades tropicales y el enfoque de género. Bol. Of. Sanit. Panam., 12: 261-81, 1996.

Ribeiro dos Santos, R.; Pirmez, C. \& Savino, W. Role of autoreactive immunological mechanisms in chagasic carditis. Res. Immunol., 142:134-7, 1991. 
Sala, M. A.; Komesu, M. C.; Lopes, R. A. \& Maia Campos, G. Karyometric study fo basal cell carcinoma. Braz. Dental J., 5:11-4, 1994.

Sala, M. A.; Matheus, M. \& Valeri, V. A new stereological method for estimating the thickness of a cellular layer on random sections. Mikroskopie, 38:127-30, 1981.

Sala, M. A.; Lopes, R. A. \& Matheus, M. Método morfológico para analisis cuantitativa de los tejidos. Determinacíon de los parâmetros normales para el hepatocito de rata. Arch. Fac. Med. Zaragoza, 32:2931, 1992.

Sala, M. A.; Matheus, M. \& Valeri, V. A stereological method for estimating average diameter on spherical structures. Braz. J. Med. Biol., 13:157-8, 1980.

Sanabria, A. Ultrastructure of Trypanosoma cruzi in mouse liver. Exp. Parasitol., 30:187-98, 1971.

Silva, J. C.; Pirmez, C.; Morgado, M. G. \& Galvão Castro, B. Immunopathological aspects of experimental Trypanosoma cruzi infection: correlation of immune complexes and other serological features with muscle lesions during the infection. Parasite Immunol., 7:45766, 1985.

Sprent, P. \& Smeeton, N. C. Applied nonparametric statistical methods. 3rd. ed. Boca Raton, Chapman \& Hall/CRC, 2001.

Tomkeieff, S. I. Linear intercepts, areas and volumes. Nature, 155:24, 1945.

Vera-Cruz, J. M.; Magallón-Gastelum, E.; Grihalva, G.; Rincón, A. R.; Ramos-García, C. \& Armendáriz-Borunda, J. Molecular diagnosis of Chagas' disease and use of an animal model to study parasite tropism. Parasitol. Res., 89:480-6, 2003.

Weibel, E. R. Stereological principles morphometry in electron microscopic cytology. Int. Rev. Cytol., 26:235302, 1969.

World Health Organization. Tropical disease research. UNDP/World Bank/W.H.O. Special programme for research and training in tropical diseases. Thirteenth programme report. World Health Organization, Geneva, Switzerland. 1997. pp. 113-23.
Dirección para correspondência:

Prof. Dr. Ruberval A. Lopes

Faculdade de Odontologia de Ribeirão Preto - USP

Av. do Cafe $s / n$

CEP 14040-904

Ribeirão Preto - SP

BRASIL

Email: ruberlopes@yahoo.com.6r

Recibido : 06-04-2006

Aceptado: 12-06-2006 